\author{
UNIVERSITY OF CALIFORNIA \\ Lawrence Radiation Laboratory \\ Berkeley, California \\ Contract No. W-7405-eng-48
}

MASTER

\title{
ANGULAR DISTRIBUTION OF FRAGMENTS FROM FISSION INDUCED BY HEAVY IONS IN GOLD AND BISMUTH
}

Victor E. Viola, Jr., T. Darrah Thomas, and Glenn T. Seaborg May 21, 1962

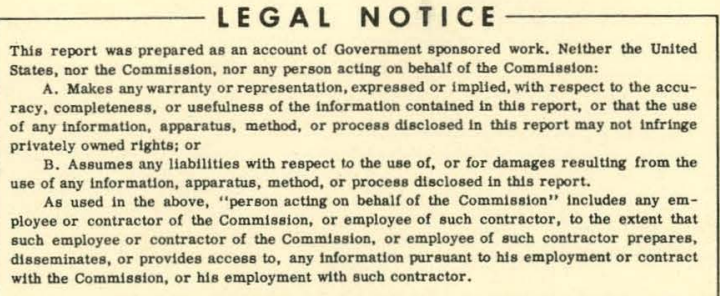




\section{DISCLAIMER}

This report was prepared as an account of work sponsored by an agency of the United States Government. Neither the United States Government nor any agency Thereof, nor any of their employees, makes any warranty, express or implied, or assumes any legal liability or responsibility for the accuracy, completeness, or usefulness of any information, apparatus, product, or process disclosed, or represents that its use would not infringe privately owned rights. Reference herein to any specific commercial product, process, or service by trade name, trademark, manufacturer, or otherwise does not necessarily constitute or imply its endorsement, recommendation, or favoring by the United States Government or any agency thereof. The views and opinions of authors expressed herein do not necessarily state or reflect those of the United States Government or any agency thereof. 


\section{DISCLAIMER}

Portions of this document may be illegible in electronic image products. Images are produced from the best available original document. 


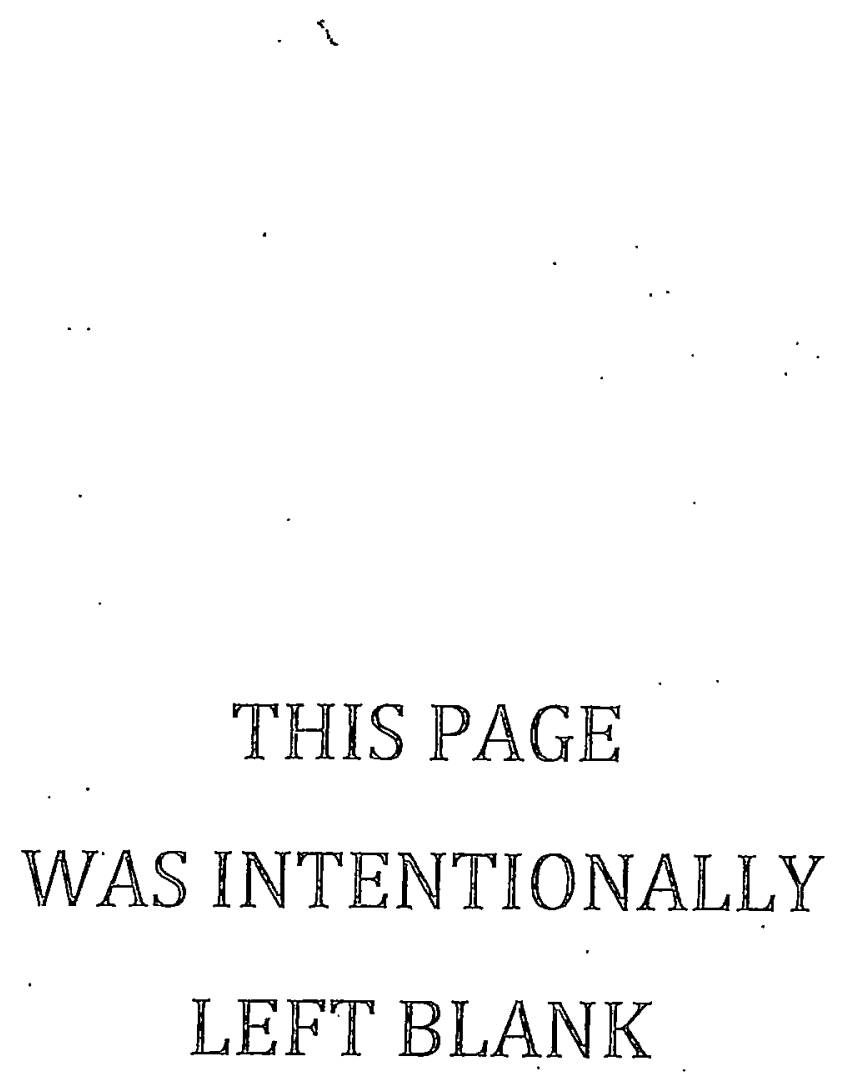




\title{
ANGULAR DISTRIBUTION OF FRAGMENTS FROM FISSION
} INDUCED BY HEAVY IONS IN GOLD AND BISMUTH

Victor E. Viola, Jr.,. T. Darrah Thomas, and Glenn T. Seaborg

\author{
Lawrence Radiation Laboratory \\ University of California \\ Berkeley, California \\ May 21, 1962
}

\section{ABSTRACT}

We present the results of measurements of the angular distribution of fission fragments produced by irradiation of $\mathrm{Au}^{197}$ and $\mathrm{Bi}^{209}$ with various heavy ions. The projectiles, $\mathrm{B}^{11}, \mathrm{C}^{12}, \mathrm{~N}^{14}$, and $\mathrm{O}^{16}$, had energies from a few MeV above the Coulomb barrier to $10.4 \mathrm{MeV}$ per nucleon. The gross features of these results can be explained by use of a model and parameters that have been used by others to account for angular distributions of fission fragments from helium - ion bombardments. In detail, however, these results appear to indicate that the models used to predict the average value of the angular momentum of the compound nucleus give values tọ low near the Coulomb barrier. Furthermore, at high bombarding energies it is necessary to consider the fact that appreciable direct interaction is taking place. 


\title{
ANGULAR DISTRIBUTION OF FRAGMENTS FROM FISSION \\ INDUCED BY HEAVY IONS IN GOLD AND BISMUTH
}

Victor E. Viola, Jr., T. Darrah Thomas, ${ }^{+}$and Glenn T. Seaborg ${ }^{\ddagger}$

\author{
Lawrence Radiation Laboratory \\ University of California \\ Berkeley, California \\ May 21, 1962
}

\section{INTRODUCTION}

The development of heavy-ion accelerators at Berkeley and at Yale has made it possible to extend the investigation of nuclear fission reactions to compound nuclei possessing large amounts of excitation energy and total angular momentum. Studies of charged-particle-induced fission at lower energies have established. that rissiun-Tragment angular distributions are related to the spin orientation and $z^{2} / A$ of the fissioning species. ${ }^{1-3}$ Heavy ions have been shown to substantially enhance these effects. ${ }^{\text {4-6 }}$

Consideration of the energy and spin states of the stably deformed nuclei led Bohr to propose a model that has been successful in explaining the anisotropies observed in low-energy fission. 7 Halpern and Strutinski ${ }^{8}$ and, independently, Griffin 9 have extended this theory to describe fission at higher energies. Interpretation of results from both heavy-ion-induced and helium-ion-induced fission studies has shown that the theory provides a reasonable model for the explanation of such reactions.

We have attempted to amplify and extend the results from earlier studies of angular distributions in heavy-ion fission. In particular we have studied differences in angular momentum, excitation energy, and $\mathrm{Z}$ among several systems. Bombardment of the monoisotopic targets $\mathrm{Au}^{197}$ and 
$\mathrm{Bi}^{309}$ with $\mathrm{B}^{11}, \mathrm{C}^{12}, \mathrm{~N}^{11}$, and $\mathrm{O}^{16}$ forms a series of compound nuclei ranging in $\mathrm{Z}$ from 84 to 91 . The cross sections for heavy-ion-induced fission for nuclei in this region are quite large, ${ }^{4,5}$ but the fission barriers are sufficiently high and the degree of excitation of the residual nuclei so low that contributions to fission from non-compound-nucleus reactions are negligible (less than $1 \%$ ). 10

\section{EXPERIMENTAL METHOD}

The data reported here were obtained by collecting the fission recoil atoms at several angles, and then measurimg their gross radioactivity. A diagram of the recoil collection chamber is shown in Fig. I. On the basis of differential recoil-range measurements as a function of angle, two techniques were adopted for catching the fission fragments. At angles of $30^{\circ}$. to the beam or less, ten $0.90-\mathrm{mg} / \mathrm{cm}^{2}$ Mylar discs were mounted in the catcher holders. This procedure usually permitted a good separation between the fission and spallation activities. In addition it provided the means for a reliable correction for activation of the catchers induced by scattered beam particles. At angles greater than $30^{\circ}$ a cover of $0.30 \mathrm{mg} / \mathrm{cm}^{2}$ or $0.90 \mathrm{mg} / \mathrm{cm}^{2}$ (depending upon angle.) was placed over a $3.30-\mathrm{mg} / \mathrm{cm}^{2}$ catcher and a $3 \cdot 30-\mathrm{mg} / \mathrm{cm}^{2}$ disc to serve as a blank. Activity in the blanks wais always negligible.

The Berkeley Hilac accelerates heavy ions to a constant terminal energy of 10.4 $\pm 0.2 \mathrm{MeV}$ per nucleon. ${ }^{11}$ Lower energies were obtained by inserting weighed beryllium foils in the beam. The energy of the degraded ions wàs determined from conversion of Northcliffe's range-energy relationships $^{12}$ for aluminum to beryllium by the use of data reported by Sternheimer. ${ }^{13}$ 


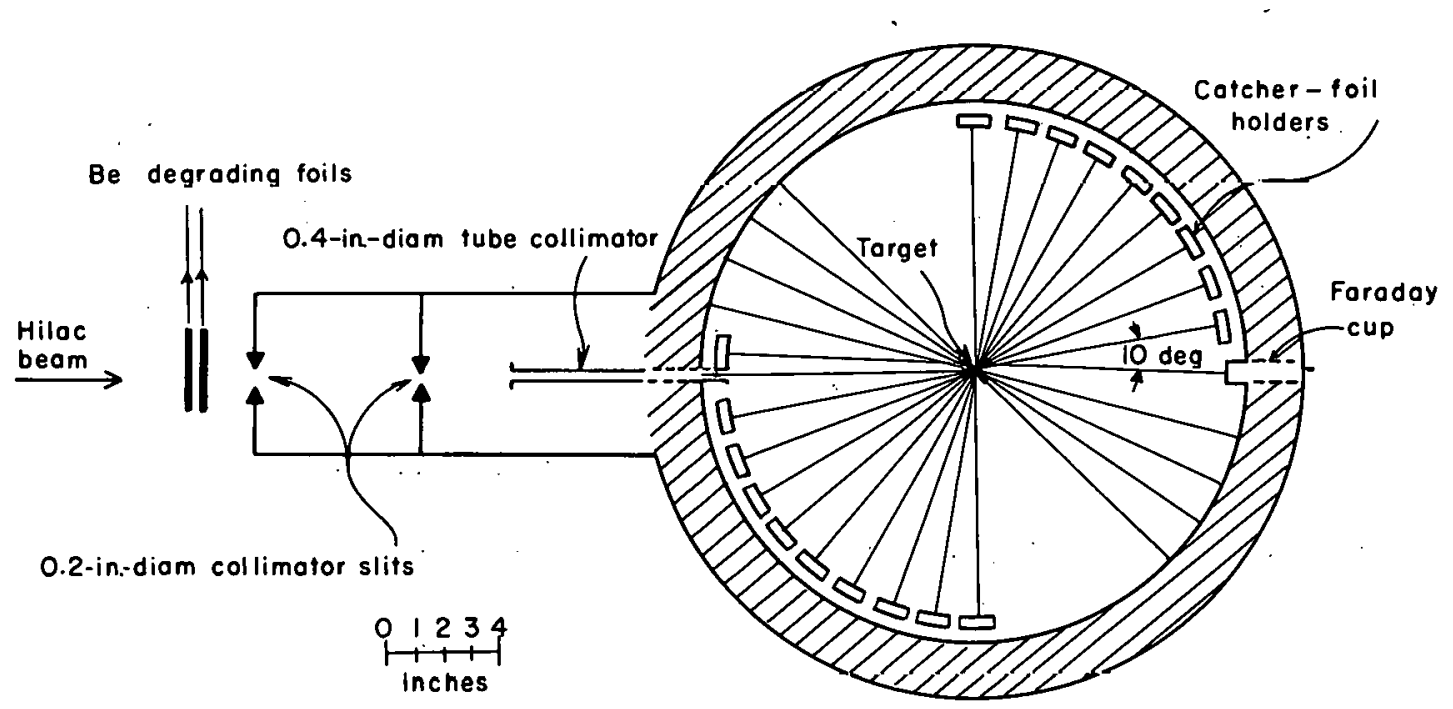

$M U-23389$

Fig. 1. Diagram of angular-distribution chamber. 
Unsupported targets of gold and bismuth ranging in thickness from about 500 to $1000 \mu \mathrm{g} / \mathrm{cm}^{2}$ were used. These target thicknesses represent but a small fraction of the fission-fragment range. For most experiments the target was oriented at $45^{\circ}$ to the beam direction. The differential cross sections did not change with variation of target angle for catchers placed at less than $60^{\circ}$ to the target normal.

The fisaion-fragment activities were measured simultaneous $\perp$ y at 32 counting stations equipped with Geiger-Mueller tubes. The register pulse from each counter was modified so that it would feed into a specific channel of a Penco 100-channel pulse-height analyzer. Counting was continued long enough to show that the angular distribution determined by gross beta counting did not depend on the time after the end of bombardment.

\section{TREATMENT OF THE DATA}

To obtain angular distributions in the laboratory (lab) system, the decay of each sample was plotted as shown in Fig. 2. Angular distributions were determined at various times during the decay in order to show the time independence of the distribution. These were then averaged to give the final lab results. From the time independence of the angular distributions and the gross decay characteristics of these decay curves, it was concluded that the experimental technique gives results that accurately represent the average f'ission process. 'l'he measurements corresponding to the bombardments with $\mathrm{N}^{14}$ and $\mathrm{O}^{16}$ have been checked further in experiments using solidstate detectors according to a system described elsewhere. ${ }^{10}$ Good agreement between the results obtained from the two techniques has been found. 


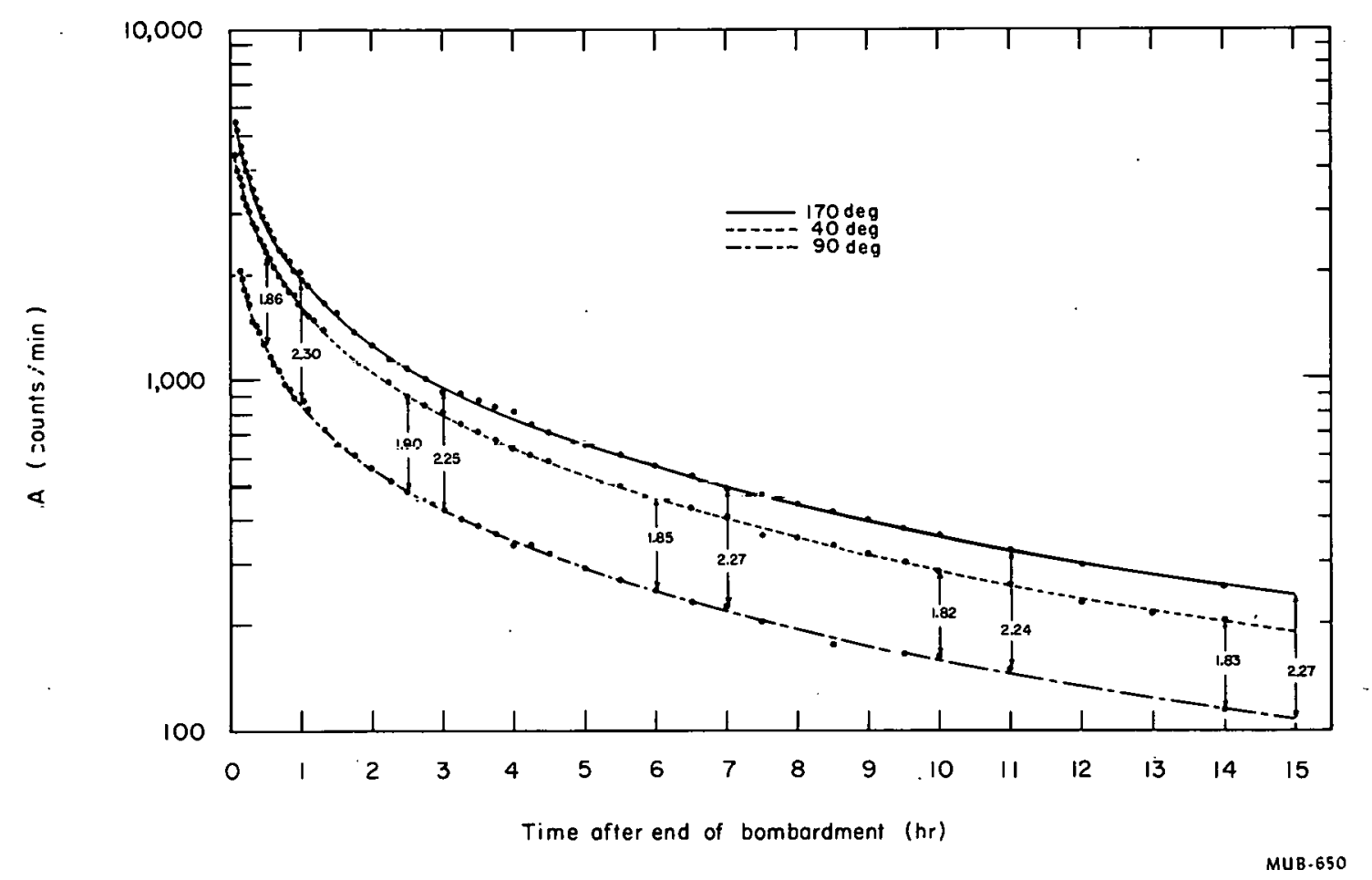

Fig. 2. Gross fission-fragment beta activity as a function of time. Limits of error on points are less than $2 \%$. 
In Fig. 3 the lab results for the system $\mathrm{Bi}^{209}+0^{16}$ are given. The error bars represent standard deviations and include errors due to counting statistics, differences in courtier geometries, and any induced activation in the catchers.

Conversion of the data into the center-of-mass (c.m.) system depends upon the quantity

$$
\mathrm{x}=\mathrm{V} / \mathrm{v},
$$

where $V$ is the velocity of the c.m. and $v$ is the velocity of the fission fragment in the c.m. system. Although a single value of $x$ cannot be rigorously applied to a manifold nuclear reaction such as fission, a most probable value for binary events, $x_{m p}$, can be estimated from the formula

$$
x_{m p}^{2}=A_{p} E_{p} A_{f} /\left(A_{C N}\right)^{2} E_{c \cdot m .}
$$

where $A_{p}$ and $E_{p}$ represent the mass and lab kinetic energy of the projectile, respectively; $A_{f}$ and $: E_{c . m}$. represent the most probable mass and c.m. kinetic energy of the fission fragment, respectively; and $A_{\text {GN }}$ represents the mass of the compound, nucleus. $A_{f}$ has been estimated from the relationship

$$
A_{f}=\frac{1}{2}\left(A_{C N}-\bar{v}\right),
$$

where $\bar{v}$ is the mean number of neutrons emitted in the fission process; $\bar{v}$ is calculated from.Leachman's result, 14

$$
\bar{v}-v_{0}+0.12 \mathrm{r}^{*},
$$




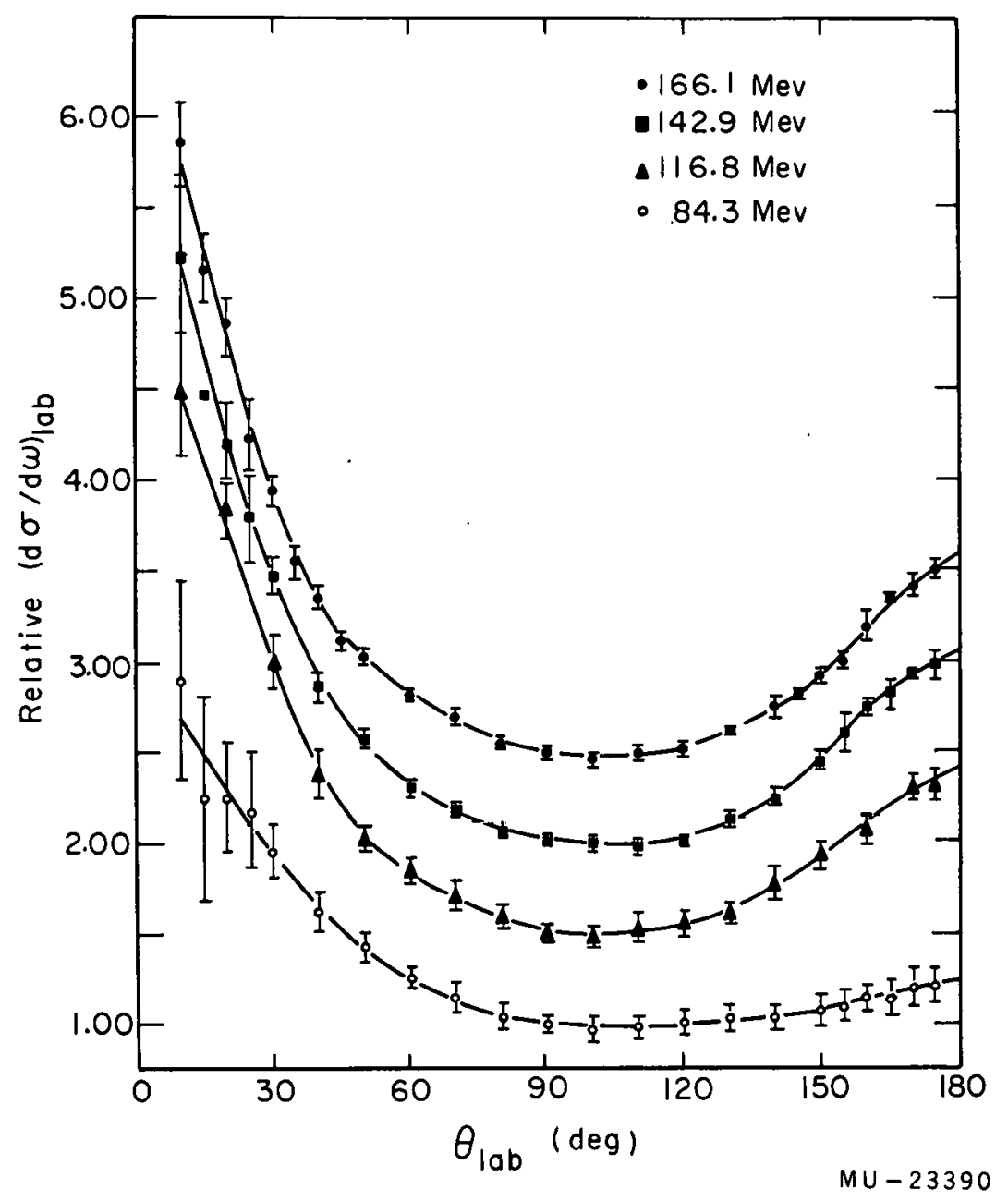

Fig. 3. Several laboratory angular distributions from $\mathrm{Bi}^{209}$ bombarded with 0l6. The errors represent standard deviations. The differential cross section at $90^{\circ}$ is unity in each case. 
where $v_{0}$ is the mean number of neutrons emitted from lile same compound nucleus undergoing spontaneous fission and $\mathrm{E}^{*}$ is the excitation energy. Values for $v_{0}$ were taken from the compilations by Huizenga and Vundenbosch, ${ }^{15}$ and $\mathrm{E}^{*}$ was calculated using the mass tables of Cameron. ${ }^{16}$ $E_{c . m}$. has been measured for many of these systems. 5,6 Unmeasured values were interpolated from the measured ones.

Values of $x_{m p}^{2}$ have also been determined by studying the angular correlation of coincident fission frugments. 10 The measured values for these systems are in good agreement with calculations based on Eq. (2). Furthermore, transformation of the data into the c.m. system with these values gives excellent symmetry about $90^{\circ}$. The transtormed angular distributions for the $\mathrm{N}^{14}+\mathrm{Au}^{197}$ system are given in Fig. 4 as a typical example.

No attempt will be made to present all the angular distributions measured. Figure 5 shows the anisotropies measured in the various systems studied, plotted as a function of the lab energy of the bombarding particle. Anisotropy is here defined to be the yield at $180^{\circ}$ divided by the yield at $90^{\circ}$, both in the center-of-mass system. Smooth curves have been drawn through the points. These results are summarized in Table I.

These curves show several obvious features. First, in every case, the anisotropy increases with increasing bombarding energy. Second, the curves for bombardment of gold targets form a group lying above a similar group of curves for bombardment of bismuth targets. Third, within each of these groups there is a progression of decreasing anisotropy with increasing projectile mass (except for the nitrogen bombardments). Similar results have been previously observed and qualitatively explained in terms of the theory presented below. ${ }^{5,6}$ Fourth, for both gold and bismuth targets 


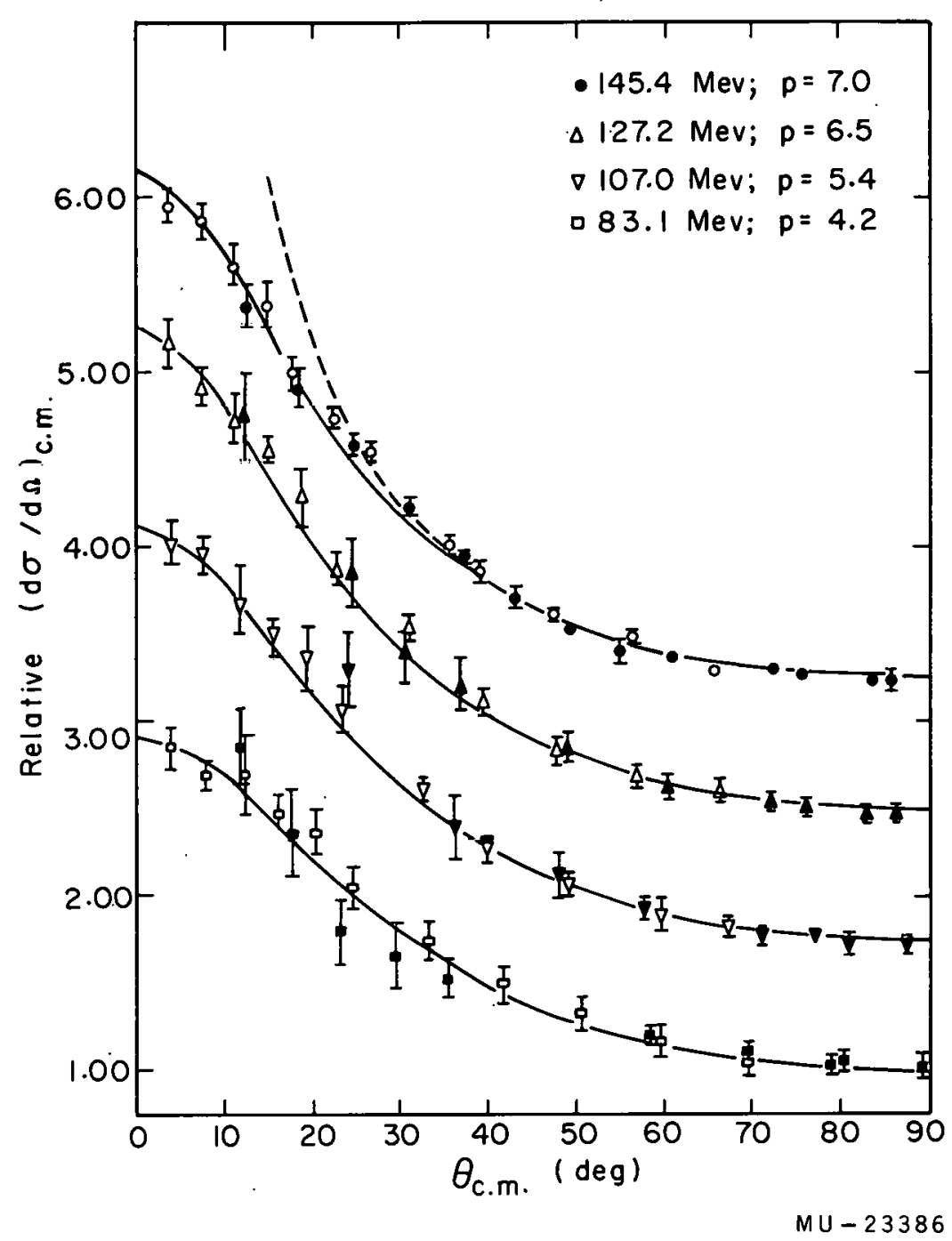

Fig. 4. Center-of-mass angular distribution from $\mathrm{Au}^{197}$ bombarded with $\mathrm{N}^{14}$. Solid curve is Halpern and Strutinski theoretical fit; broken curve is plot of $1 / \sin \theta$. The differential cross section at $90^{\circ}$ is unity. (Solid points refer to catcher angle $\theta$; open points to $\pi-\theta$.) 

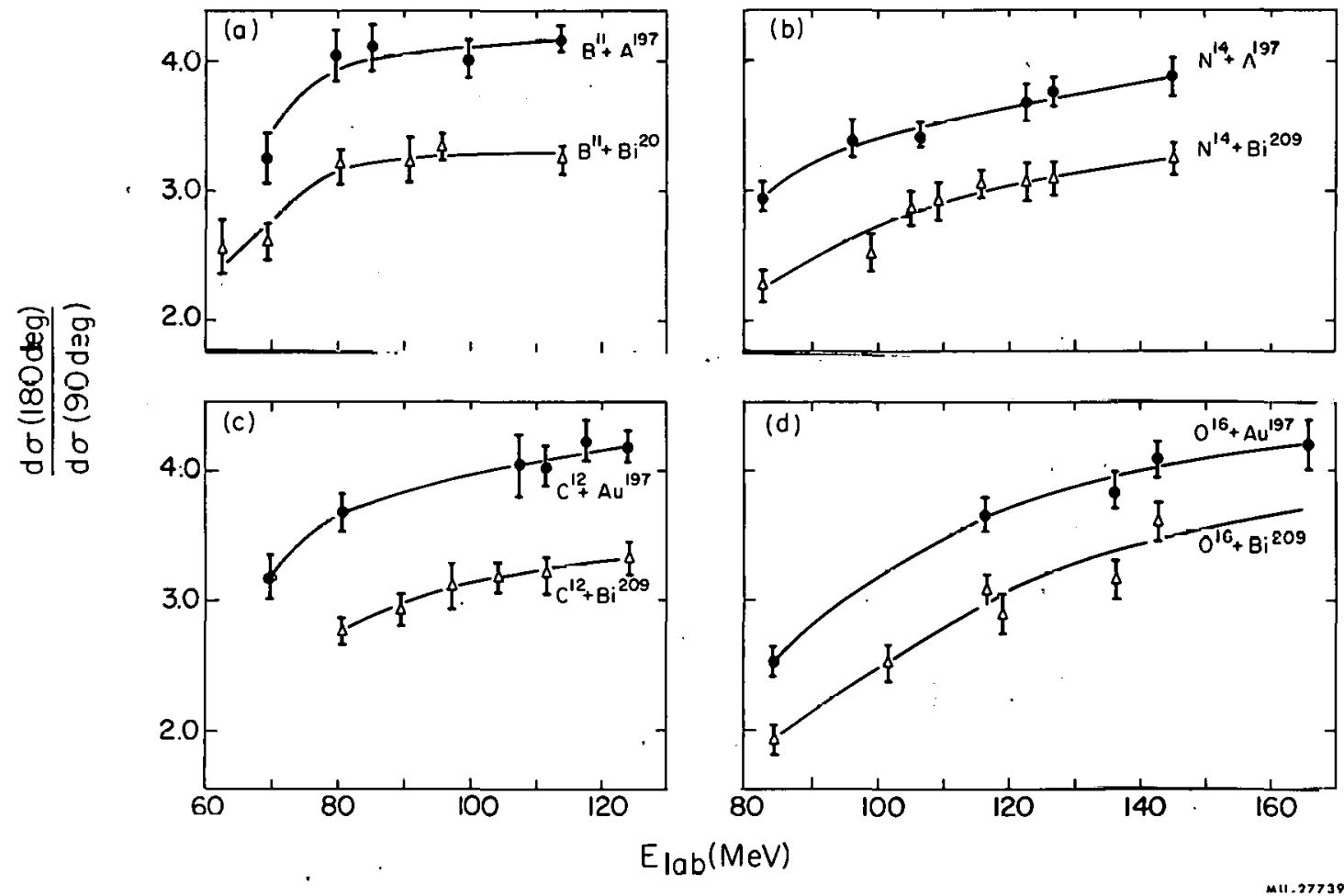

Fig. 5. Variation of the center-of-mass anisotropy with bombarding energy for (a) $\mathrm{B}^{11}$, (b) $\mathrm{C}^{12}$, (c) $\mathrm{N}^{14}$ and (d) 016 incident upon both $\mathrm{Au}^{197}$ and $\mathrm{B} 1209$ targets. 
Table I. Measured and calculated properties of each system studied here. Symbols are defined in the text.

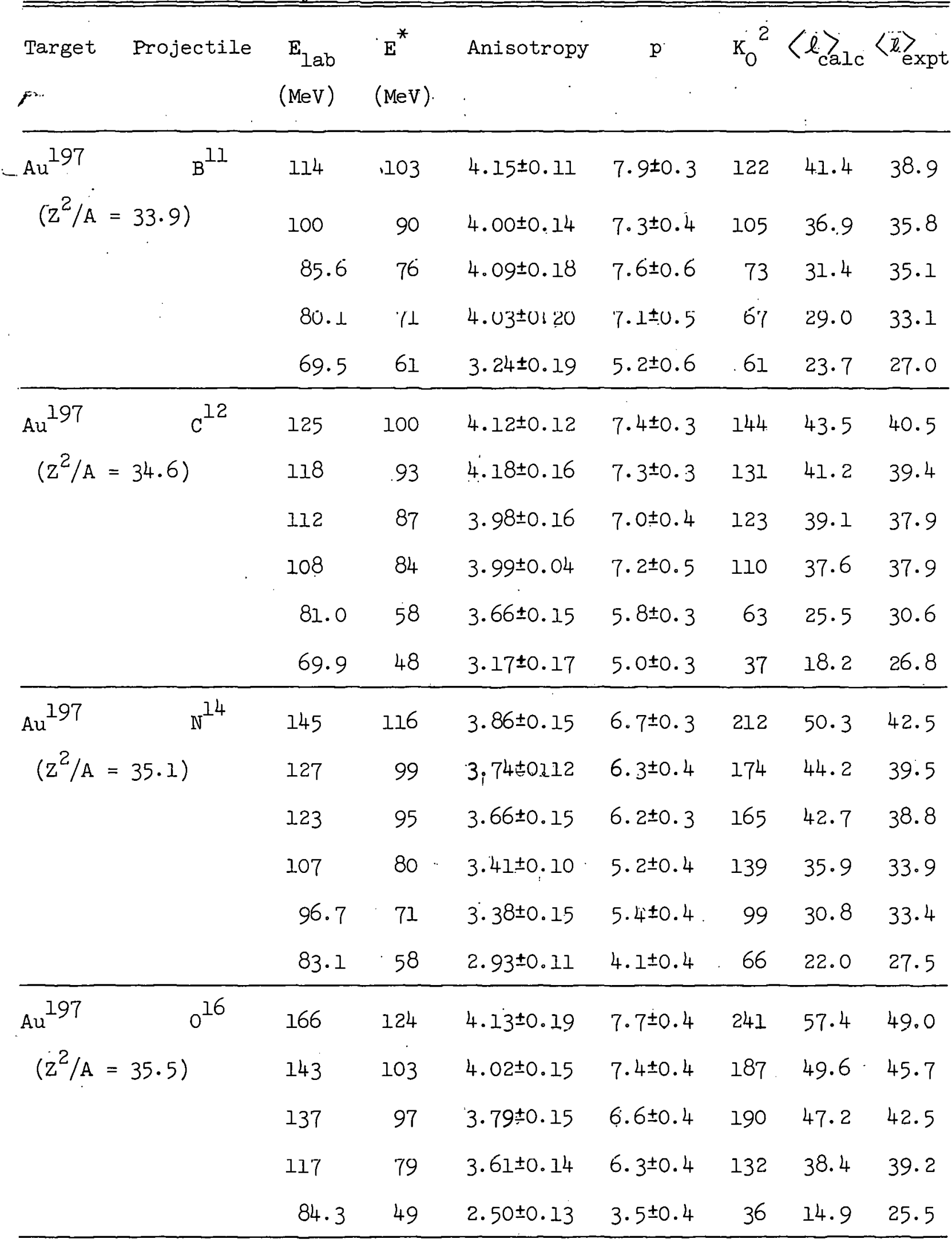


l'áble I. (Cont.)

\begin{tabular}{|c|c|c|c|c|c|c|c|c|}
\hline Target & Projectile & $\begin{array}{l}E_{1 a b} \\
(\mathrm{MeV})\end{array}$ & $\begin{array}{c}\mathrm{E}^{*} \\
(\mathrm{MeV})\end{array}$ & Anisotropy & $\mathrm{p}$ & $\mathrm{K}_{\mathrm{O}}^{2}$ & écalc & $e \sum_{\text {expt }}$ \\
\hline \multirow[t]{6}{*}{$\mathrm{Bi}^{209}$. } & $\mathrm{B}^{11}$ & 114 & 89 & $3 \cdot 32 \pm 0.11$ & $5 \cdot 3 \pm 0.3$ & 185 & 41.7 & $37 \cdot 5$ \\
\hline & $=35.2)$ & $95 \cdot 9$ & 71 & $3.22 \pm 0.13$ & $5.1 \pm 0.3$ & 137 & $35 \cdot 3$ & 34.8 \\
\hline & & 91.0 & 67 & $3.20 \pm 0.16$ & $5.0 \pm 0.3$ & 126 & $33 \cdot 4$ & $33 \cdot 7$ \\
\hline & & 80.4 & 57 & $3.17 \pm 0.14$ & $4.8 \pm 0.3$ & 97 & 28.8 & 31.6 \\
\hline & & 69.5 & 46 & $2.60 \pm 0.14$ & $3.5 \pm 0.3$ & 87 & 23.2 & 25.6 \\
\hline & & 62.7 & 40 & $2.62 \pm 0.21$ & $3 \cdot 3 \pm 0.5$ & 58 & 18.5 & 23.8 \\
\hline \multirow{6}{*}{$\begin{array}{l}\mathrm{Bi}^{209} \\
\left(\mathrm{z}^{2} / \mathrm{A}\right.\end{array}$} & $c^{12}$ & 125 & 85 & $3.27 \pm 0.14$ & $5 \cdot 3 \pm 0.3$ & 201 & $43 \cdot 5$ & 39.7 \\
\hline & $=35.8)$ & 112 & 73 & $3.15 \pm 0.14$ & $4.9 \pm 0.3$ & 175 & 39.0 & 36.7 \\
\hline & & 105 & 66 & $3.14 \pm 0.11$ & $4.6 \pm 0.3$ & 161 & 36.3 & $34 \cdot 5$ \\
\hline & & 97.4 & 59 & $3.07 \pm 0.17$ & $4.4 \pm 0.3$ & 140 & $33 \cdot 1$ & 32.9 \\
\hline & & $89 \cdot 7$ & 52 & $2.90 \pm 0.12$ & $3 \cdot 9 \pm 0.3$ & 125 & $29 \cdot 5$ & 29.9 \\
\hline & & 87.0 & 44 & $2.74 \pm 0.10$ & $3.6 \pm 0.3$ & 95 & 24.7 & $27 \cdot 4$ \\
\hline \multirow{8}{*}{$\begin{array}{l}\mathrm{B}^{209} \\
\left(\mathrm{z}^{2} / \mathrm{A}\right.\end{array}$} & $\mathrm{N}^{14}$ & 145 & 101 & $3.23 \pm 0.15$ & $4.9 \pm 0.3$ & 292 & 50.4 & 42.2 \\
\hline & $=36 \cdot 3)$ & 127 & 84 & $3.07 \pm 0.13$ & $4.5 \pm 0.3$ & 24,2 & 44.0 & 38.6 \\
\hline & · & 12.3 & 81 & $3.04 \pm 0.15$ & $4.5 \pm 0.3$ & 225 & 42.4 & $38 . ?$ \\
\hline & & 116 & 74 & $3.03 \pm 0.10$ & $4.5 \pm 0.3$ & 195 & $39 \cdot 5$ & $37 \cdot 3$ \\
\hline & & 110 & 68 & $2.90 \pm 0.15$ & $4.1 \pm 0.3$ & 185 & 36.8 & $35 \cdot 1$ \\
\hline & & 106 & 65 & $2.84 \pm 0.14$ & $3 \cdot 9 \pm 0.3$ & 176 & 34.9 & $33 \cdot 7$ \\
\hline & . & $99 \cdot 5$ & 59 & $2.50 \pm 0.14$ & $3.6 \pm 0.3$ & 155 & $31 \cdot 5$ & 31.5 \\
\hline & & 83.1 & 43 & $2.24 \pm 0.12$ & $2.5 \pm 0.3$ & 94 & 20.4 & 24.4 \\
\hline
\end{tabular}


Table I. (Cont:)

\begin{tabular}{|c|c|c|c|c|c|c|c|c|}
\hline \multirow{2}{*}{$\frac{\text { Target }}{\mathrm{Bi}^{209}}$} & Projectile & $\begin{array}{l}E_{\text {lab }} \\
(\mathrm{MeV})\end{array}$ & $\begin{array}{c}E^{*} \\
(\mathrm{MeV})\end{array}$ & Anisotropy & $\mathrm{p}$ & \multicolumn{2}{|c|}{$\mathrm{K}_{0}^{2}\langle\ell\rangle_{\mathrm{Calc}}$} & e expt \\
\hline & $0^{16}$ & 166 & 107 & $3.65 \pm 0.13$ & $6.0 \pm 0.3$ & 309 & 57.4 & 49.2 \\
\hline \multirow{2}{*}{\multicolumn{2}{|c|}{$\left(z^{2} / A=36.8\right)$}} & 143 & 86 & $3.55 \pm 0.20$ & $5.8 \pm 0.5$ & 235 & 49.2 & 45.7 \\
\hline & & 137 & 80 & $3.11 \pm 0.15$ & $4.7 \pm 0.3$ & 261 & 46.7 & 40.5 \\
\hline \multirow{4}{*}{\multicolumn{2}{|c|}{ : }} & 119 & 64 & $2.85 \pm 0.15$ & $4.0 \pm 0.3$ & 209 & 38.6 & 35.2 \\
\hline & & 117 & 62 & $3.03 \pm 0.11$ & $4: 5 \pm 0.3$ & 176 & $37 \cdot 5$ & 37.0 \\
\hline & & 102 & 48 & $2.48 \pm 0.15$ & $3 \cdot 1 \pm 0.4$ & 147 & 28.5 & 28.8 \\
\hline & & $84 \cdot 3$ & .32 & $1.89 \pm 0.11$ & $1.7 \pm 0.3$ & 46 & 11.8 & $19 \cdot 3$ \\
\hline
\end{tabular}


the anisotropies measured in the nitrogen bombardments tend to tall low with respect to the sequence just mentioned. This anomaly most probably has its origin in nuclear surface reactions which, as will be discussed later, apparently are an important consideration in heavy-ion fission studies. Similar plots can be made of anisotropy versus excitation energy, average orbital angular momentum brought in by the projectile, or kinetic energy in excess of the Coulomb barrier. Although such plots differ in detail from those shown in Fig. 5, they are qualitatively the same.

\section{INTERPRETATION OF RESULTS}

\section{A. Theory}

According to Bohr, the quantum states of the fissioning nucleus at the saddle point are described by $I$, the total angular momentum; $K$, the projection of $I$ on the nuclear symmetry axis; and $M$, the projection of $I$ along the beam direction. 7 The angular distribution is then described by the square of the symmetric top wave function $\left|D_{M K}^{I}\right|^{2}$.

For fission induced by a beam of particles, $M$ is no greater than the spin of the target nucleus, and $I$ is approximately equal to the orbital angular momentum, $\ell$, of the incident particle. If the average value of $\ell,\langle\ell$, is sufficiently large, it is possible to assume $\mathrm{M}=0$ and to replace the symmetric top wave functions by the classical expression

$$
\begin{aligned}
\left|D_{O K}^{I}\right|^{2} & \propto\left[\sin ^{2} \theta-\left(K^{2} / I^{2}\right)\right]^{-I / 2}, \\
& =0 \text { for } \sin \theta>K / I \\
& \text { for } \sin \theta \leqslant K / I
\end{aligned}
$$


Averaging over the respective distributions in $I$ and $K, G(I)$ and $F(K)$, Bohr obtains the angular distribution

$$
W(\theta) \int_{0}^{I \max } d I \int_{I \leqslant}^{K} \sin \theta d K G(I) F(K) /\left(\sin ^{2} \theta-K^{2} / I^{2}\right)^{I / 2} .
$$

Both Halpern and Strutinski, and Griffin, assume that $G(I)$ should be well approximated by the classical distribution of spin states. 8,9 For $F(K)$ Halpern and Strutinski propose a Gaussian distribution based on statistical theory. The anisotropy predicted by this treatment is characterized by the parameter

$$
p=\frac{I_{\text {max }}^{2}}{4 K_{0}^{2}}=\frac{\hbar^{2}}{2 T} \frac{I^{2} \max }{2 T}
$$

where $K_{0}$ is the mean value of $K, \$_{\text {eff }}$ is the effective moment of inertia of the nucleus at the saddle point, and $\mathrm{T}$ is the nuclear temperature. The effective moment of inertia is defined as

$$
\frac{1}{\operatorname{sif}_{\text {eff }}}=\frac{1}{11}-\frac{1}{1}
$$

The quantity 11 is the moment of inertia with respect to the symmetry axis and 1 is the moment of inertia with respect to an axis perpendicular to the symmetry axis. In this detinition eff decreases as the elongation of the nucleus increases. 


\section{B. Application of the Theory}

Chaudhry, Vandenbosch, and Huizenga have successfully applied this theory to analysis of angular distributions of fragments from fission induced by helium ions in various targets. ${ }^{3}$ According to their treatment,

$$
T=\sqrt{\left(E^{*}-E_{\text {th }}-E_{\text {rot. }}\right) / a}
$$

where $E^{*}$. is the excitation energy of the fissioning nucleus, $E_{t h}$ is the height of the fission barrier, $E_{\text {rot }}$ is the rotalional energy of the rucleus at the saddle point, and $a$ is the usual level-density parameter. on the basis of other experiments ${ }^{17}$ they have developed an empirical relationship. for predicting $E_{t h}$ and have shown that a reasonable value of a is $\mathrm{A} / 8 \mathrm{MeV}^{-1}$. Using these parameters and values of $I_{\max }^{2}$ based on the opticalmodel calculations by Huizenga and Igo $^{18}$ they have been able to interpret their angular distribution data on the basis of an effective moment of inertia, eff , that decreases with decreasing. $z^{2} / A$.

We have attempted to analyze our data in the same way: . We first make the assumption that fission is occurring in the original compound nucleus. It is quite possible that this assumption is wrong; we investigate its implications in appendix A.

In estimating the excitation energy above. the saddle point, we have calculated the total excitation energy, $E^{*}$, from the mass table of Everling, Konig, Wapstra, and Mattauch (EKWM) ${ }^{19}$ (except for the oxygen bombardments, for which it was necessary to use Cameron's mass table. ${ }^{16}$ ). The fission barrier heights, $\mathbf{E}_{t h_{1}}$, were based on the formula of Huizenga, Chaudhry, and Vandenbosch, 17 again using the EKWM mass tables where possible and pairing and shell corrections from Cameron where not. The energy tied up in rotational 
motion, $E_{\text {rot }}$, was taken to be $\langle l\rangle^{2} \hbar^{2} / 2 s_{0}$, where $\Phi_{0}$ is the rigid-body moment of inertia of a sphere and $\langle\ell\rangle$ is the average orbital angular momentum brought in by the heavy ion. The method for estimating this latter quantity is described below. Strictly speaking, we should use eff rather than $\$_{0}$, but since $W_{\text {eff }}$ is not much different from $\$_{0}$ and since $E_{\text {rot }}$ is invariably small compared with $E^{*}$, no appreciable error is introduced by this assumption. In the appendix we discuss in more detail the choice of moment of inertia.

The quantity $I_{\max }^{2}$ was assumed to be equal to $9 / 4\langle\ell\rangle^{2}$. The average value of the orbital angular momentum, $\langle\ell\rangle$, was calculated by using the parabolic approximation to the real part of the optical-model potential described by Thomas. ${ }^{20}$ The parameters used in these calculations (i.e., nuclear potential-well depth, nuclear surface thickness, and nuclear radius) were those necessary to give the correct values for the total reaction cross section for the systems $\mathrm{U}^{238}$ plus $\mathrm{B}^{11}, \mathrm{C}^{12}, \mathrm{~N}^{14}$, and $\mathrm{O}^{16}$, as measured by Viola and Sikkeland. 21

All the information needed to calculate values of eff was thus available. The results of such calculations are shown in Fig. 6, where we have plotted eff ${ }_{0}$ versis $Z^{2} / A$. Also shown in this graph are the data of Chaudhry et al. 3 who found $\$_{\text {eff }}$ to be independent of excitation energy. A smooth curve connects their five points. We note that, although the points based on our work bracket this smooth curve, the range of deviation is quite large. However, these deviations are systematic: the points falling above the curve correspond to bombardments at the highest energies, whereas points falling below the curve correspond to bombardments at the lowest energies. In particular, the three points falling the farthest below the curve are from bombardments at energies only a few MeV above the Coulomb barrier. 


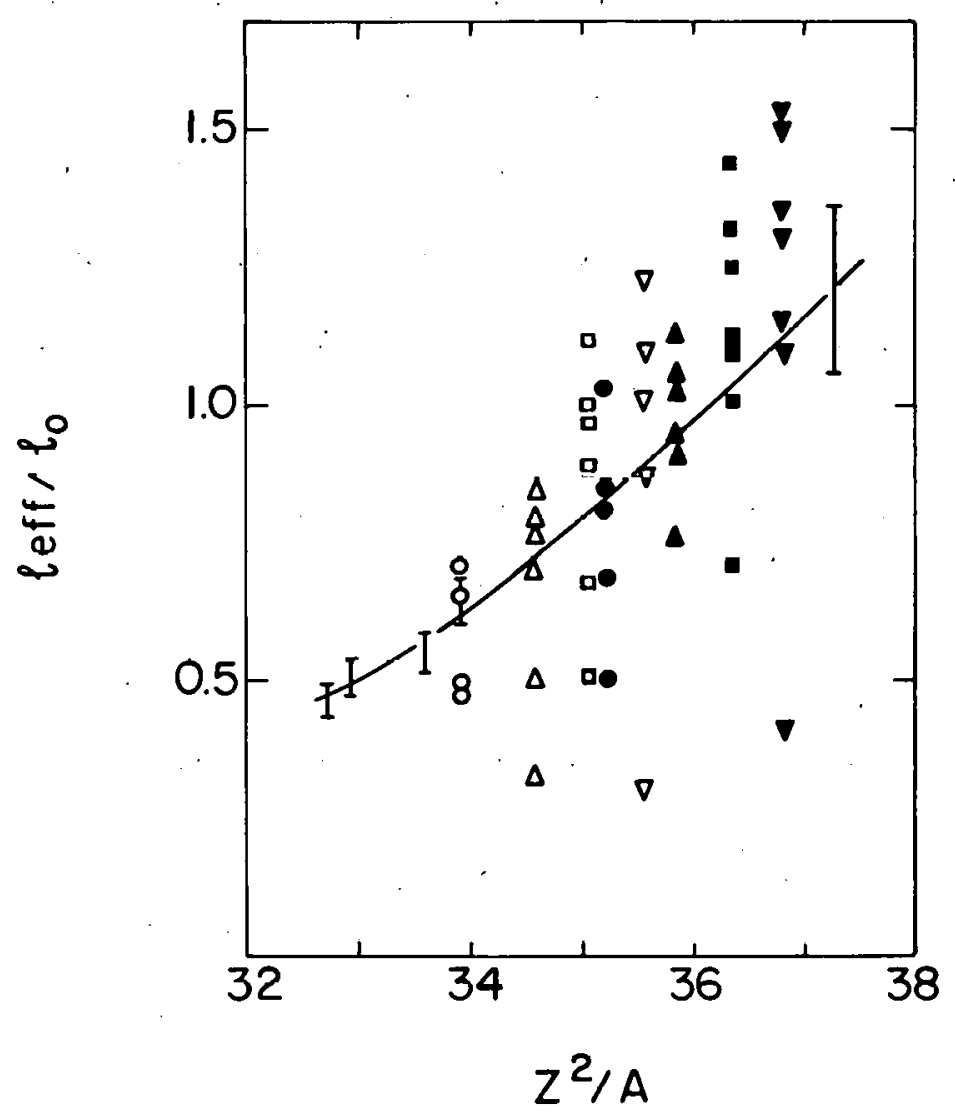

$M U-27738$

Fig. 6. Ratio of effective moment of inertia at the saddle point, $\Im_{\text {eff }}$, to the moment of inertia of a rigid sphere, $\widetilde{\Im}_{0}$, plotted against $x=\frac{Z^{2} / A}{50.13}$ for various fissioning systems. Open points refer to bombardments of $\mathrm{Au}^{197}$, closed to bombardments of $\mathrm{Bi}^{209}$. Bombarding projectiles are indicated

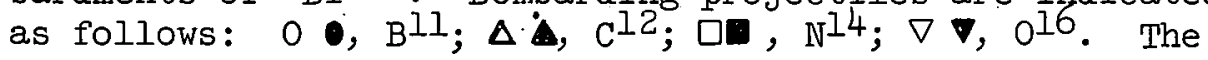
vertical lines are from Ref. 3. The line is a smooth curve connecting the points from Ref. 3 . 
The most reasonable explanation of this behavior is that we are not predicting the correct value of $\langle\ell\rangle$. To investigate this possibilitty, we have assumed that ${ }_{\text {eff }} / \$_{0}$ is given by the curve shown in Fig. 6 and then calicuiated the values of $\langle\ell\rangle$ necessary to give this moment of inertia from the experimental data. Designating this quantity as $\langle l\rangle$ expt' we plot the ratio of $\langle\ell\rangle_{\text {expt }}$ to $\langle\ell\rangle_{\text {calc }}$, as calculated from the parabolic approximation, versus the kinetic energy of the bonburding particle above the Coulomb barrier. We see in Fig. 7 that all the data for the eight different systems fall rough $\perp$ y on one curve. 'l'his result seems to support the idea that we are calculating $\langle\ell\rangle$ badly, and that we are (a) underestimating it near the barrier and (b) overestimating it at high energies.

It is not surprising that the model used for the calculation of $\langle l\rangle$ does not give good results near the Coulomb barrier. That this should be the case has been pointed uut by Hulzenga and Igo. 18 In anaiyzing the tọtal reaction cross sections for heavy-ion bombardment of $\mathrm{U}^{238}$, Viola and sikkeland encountered difficulty in obtaining a consistent fit between those determined at the maximum and minimum energies and that determined at 5 to $15 \mathrm{MeV}$ above. the Coulomb barrier. ${ }^{21}$ However, such improvements as one might make in the model would give still smaller values of $\langle l\rangle$ at low energies. Calculations based on a square-well model lead to essentially the same results unless we use an $r_{0}$ value of about 1.8 fermis.

Aside from the possibility that the model for calculating $\langle\ell\rangle$ is wrong, there are two effects that might make the average angular momentum of the fissioning nucleus different from that calculated. These are the effects of direct interactions and the effect of competing compound-nucleus reactions.

Sikkeland and Viola have investigated direct interactions in heavyion-induced reactions and estimate that in the system $\mathrm{U}^{238}+166-\mathrm{MeV} \mathrm{O}^{16}$ ions, 


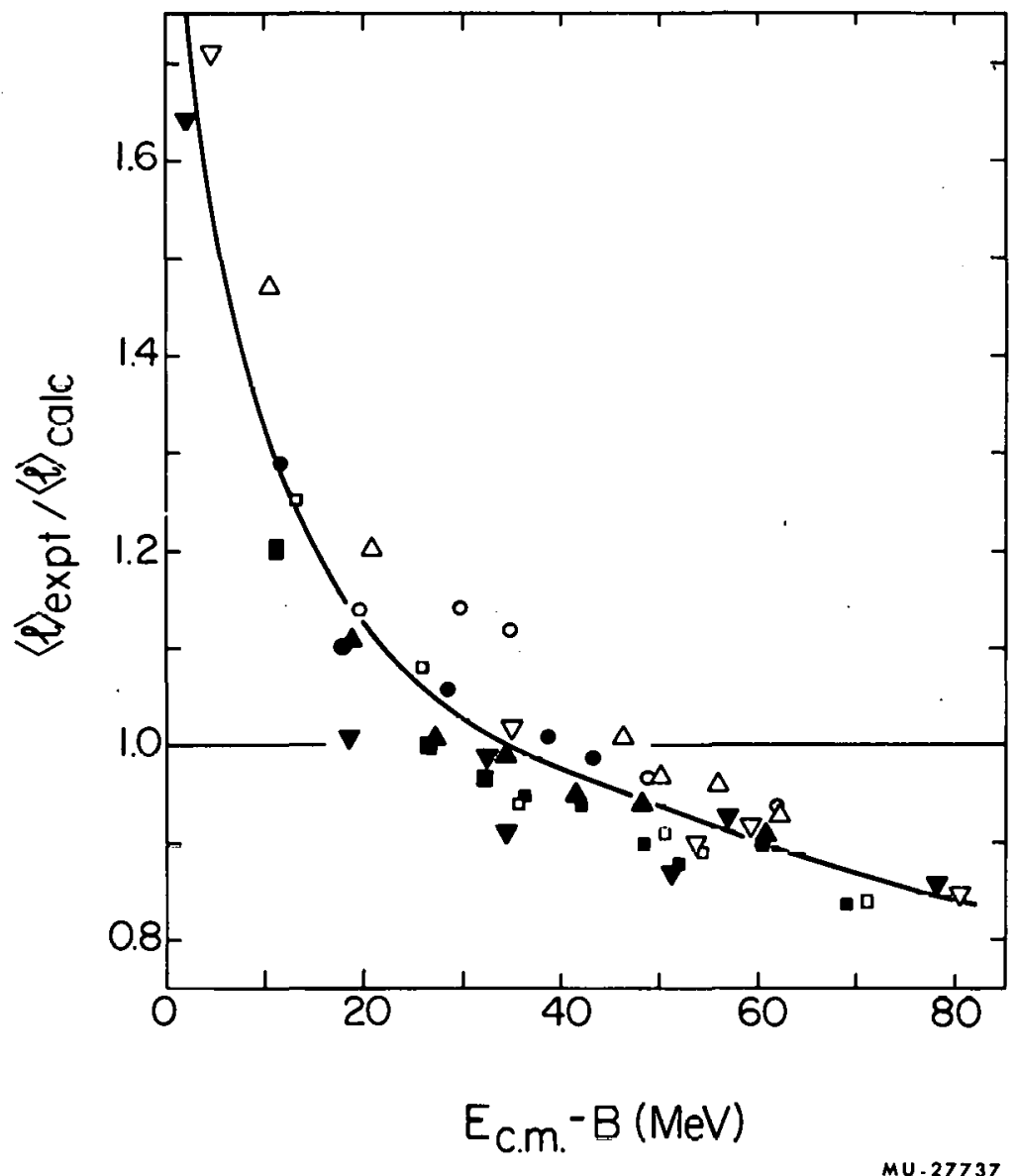

Fig. 7. Ratio of experimental to calculated average anglilar momentum plotted against center-of-mass kinetic energy of the projectile. in excess of the Coulomb barrier. The various symbols have the same significance as in Fig. 6. 
approximately $25 \%$ of the reaction cross section involves direct interaction. ${ }^{22}$

Further, their work indicates that in systems such as $\mathrm{Bi}^{209}+\mathrm{O}^{16}$ essentially none of the direct interactions leads to fission. Presumably these. direct interactions are surface reactions and occur at the expense of the formation of compound nuclei with high spin states. To illustrate this effect, we assume that in the system $\mathrm{Bi}^{209}+166-\mathrm{MeV} \mathrm{O}^{16}$ ions $25 \%$ of the reaction cross section gues inlu alrect Interactions removing the highest $l$ waves from the compound-nucleus-formation cross section. We assume further that all the compound nuclei formed undergo fission. The total reaction cross section is calculated from the parabolic approximation to be $2160 \mathrm{mb}$ with $\langle\ell\rangle=57.4$. Correcting these for the $25 \%$ of the reactions in which no compound nucleus is formed, we get a fission cross section of $1620 \mathrm{mb}$ to be compared with a value measured by Britt and Quinton of $1630 \mathrm{mb} .^{6}$ The corrected value of $\langle\ell\rangle$ is 49.4 , to be compared with $\langle\ell\rangle$ expt of 49.2 . This agreement is rather encouraging.

For the system $\mathrm{Au}^{197}+\mathrm{C}^{12}$ it is known that not all the compound nuclei formed undergo fission. 23 some de-excite by neutron emission to give astatine isotopes. If we assume that fissionability increases with increasing angular momentum, we conclude that the products that survive the competition from fission must in general have low angular momentum. Hence we might expect the actual value of $\langle I\rangle$, the average value of the angular momentum, for the fissioning nuclei to be somewhat higher than $\langle\ell\rangle$ for all the compound nuclei. To illustrate this effect, we consider the case of $69.5-\mathrm{MeV}$ carbon ions incident on $\mathrm{Au}^{197}$. Since this energy is not far above the Coulomb barrier, we may be safe in assuming that there is no direct interaction, ${ }^{24}$ although the dependence of surface reactions on bombarding energy is not completely resolved. 
The calculated reaction cross section is $677 \mathrm{mb}$; the fission cross section measured by Gordon et al. ${ }^{5}$ at this energy is $100 \mathrm{mb}$. If we assume that only the highest angular momentum states fission, we calculate $\langle I\rangle=26.2$; this is to be compared with a value of $\langle\ell\rangle$ expt of 26.8 .

This agreement not only appears to be too good to be true, it actually is. Classically, the maximum value of $I=3 / 2\langle\ell\}$. Hence, using this approach we can ncver find a value of $I>3 / 2\langle\ell\rangle$. However, we t'znd two cacer with $\left\langle p_{1}\right\rangle_{\text {expt }}\left|\left\langle p_{1}\right\rangle_{\text {calc }}\right\rangle$ 1.5. Furthermore, one of these is the case of $\mathrm{O}^{16}+\mathrm{Bi}^{209}$, where we might expect from the systematics of the competition between fission and neutron emission that all the nuclei would eventually f'ission. 15

However, in spite of the failure to obtain complete agreement between experiment and theory, we can summarize the situation by noting that if we make alloweance for a reasonable amount of direct interaction at high bombarding energies and for an inability to predict values of $\langle\ell\rangle$ for bombarding energies near the Coulomb barrier, the data are consistent with the theory. Speaking more quantitatively, all but five of the 49 data carl be accounted for by values of $\langle l\rangle$ differing by not more than $20 \%$ from the predicted ones and values of all other parameters taken from other experimental results. 


\section{VIT. DISCUSSION}

Chaudhry et al ${ }^{3}$ have discussed the moments of inertia in terms of two models: one in which the nucleus at the saddle point is shaped like a spheroid; the other in which it is shaped like two equal spheroids in contact, with symmetry about the axis connecting their centers of mass. Neither their data nor ours provide any means for distinguishing between these two models. One would expect that for $\mathfrak{\Im}_{\text {eff }} / \mathfrak{\Im}_{0}>0.9$ the two-spheroid model. would not be applicable, since in this case the two spheroids would be oblate. However, regardless of which model is chosen, both their experiments and ours indicate that as $\mathrm{z}^{2} / \mathrm{A}$ decreases the deformation at the saddle point increases. For low values of $\mathrm{z}^{2} / \mathrm{A}$ the saddle-point configuration is extremely elongated, with the extension of the nucleus along the axis of symmetry being perhaps four or five times its extension perpendicular to that axis. Cohen and Swiatecki have proposed that there is a rapid change in the sequence of liquid-drop saddle-point shapes for nuclei with fissionability parameter $\mathbb{x}$ in the neighborhood of $\mathbb{x}=0.7 .^{26}$ According to their interpretation; this rapid change should result in a rapid increase in the elongation of the fissioning nucleus as $\mathbb{X}$ decreases across this region. The $\mathbb{X}$ values for the compound nuclei we have studied vary from about 0.68 to 0.735 . Thus, our data agree qualitatively with the liquid-drop-model calculations.

In spite of the difficulties of interpreting experiments done with heavy ions, these results together with those of Chaudhry et al. suggest that it might be interesting to investigate angular distribution of fission fragments using targets substantially lighter than gold, such as the rare earths. 


\section{ACKINOWLEDGMENTS}

We wish to thank Dr. John M. Alexander for many helpful discussions concerning this work. Also we are indebted to Dr. Edward I. Hubbard for permitting us to use his counting equipment during these experiments. We would like to acknowledge the assistance of $\mathrm{Mr}$. Charles $\mathrm{A}$. Corum, who helped in designing the apparatus, and of $\mathrm{Mr}$. Daniel A. O'Connell, who prepared the targets. 


\section{APPENDIX}

In this section we discuss the assumption that fission takes place before neutron emission, and the choice of the moment of inertia of the fissioning nucleus.

\section{A. Effects of Neutron Emission.}

The nuclear parameter of interest that we obtain from studies of angular distribution of fission fragments is the quantity $\mathfrak{\Im}_{\text {eff }} / \widetilde{s}_{0}$, the ratio of the effective moment of inertia at the saddle point to the rigidbody moment of a sphere. From Halpern and Strutinski's work we can say

$$
\Im_{\text {eff }} / \Im_{n}=\hbar^{2} K_{n}^{2} / T_{n} \text {. }
$$

If we assume that neutrons are emitted before fission we must use different values of $T$ and $\mathfrak{J}_{0}$ from those used above. To evaluate this effect, consider the logarithmic derivative of $\mathfrak{s}_{\text {eff }} / \mathfrak{s}_{0}$ with respect to mass number A,

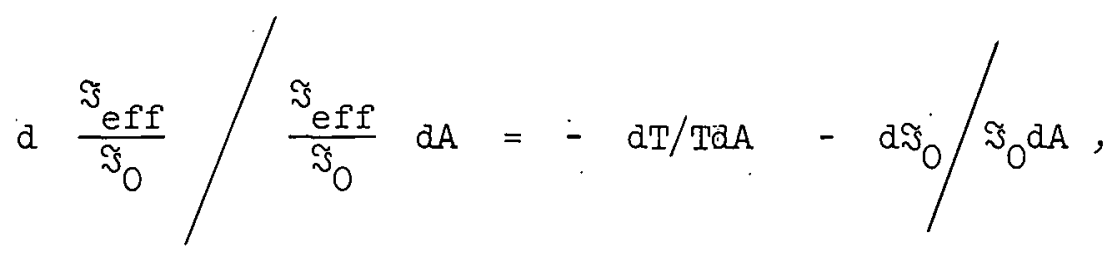

$$
\begin{aligned}
& \frac{d T}{T d A}=\frac{d T}{T d E^{*}} \frac{d E^{*}}{d A} .
\end{aligned}
$$


We assume that the energy loss on emission of one neutron is $10 \mathrm{MeV}$ and that $T=\left(\mathrm{E}^{*} / \mathrm{a}\right)^{\mathrm{l} / 2} ;$ then,

$$
\mathrm{dT} / \mathrm{TdA}=5 / \mathrm{E}^{*}
$$

The moment of inertia, $\Im_{0}$, is given by the relationship

$$
\begin{aligned}
& \mathfrak{\Im}_{0}=(2 / 5) \mathrm{AR}^{2}, \\
& \mathfrak{\Im}_{0}=(2 / 5) \mathrm{r}_{0}^{2} \mathrm{~A}^{5 / 3} \text { for } \mathrm{R}=\mathrm{R}_{0} \mathrm{~A}^{\mathrm{I} / 3} .
\end{aligned}
$$

Thus

$$
\mathrm{d} \mathfrak{J}_{0} \mathfrak{\Im}_{1} \mathrm{dA}=(5 / 3) \mathrm{A}^{-1}
$$

Since A is approximately 200 for the nuclei under consideration, this. last term is of the order of 0.01 .

Hence

$$
\text { a. } \frac{\widetilde{s}_{\text {eff }}}{\widetilde{s}_{0}} / d \mathrm{dA}=-\frac{\tilde{\Im}_{\text {eff }}}{\widetilde{s}_{0}}\left(\frac{4}{F_{*}}+0.01\right)
$$

Furthermore, if we assume that neutrons are emitted prior to fission, the fissionability parameter $\mathrm{Z}^{2} / \mathrm{A}$ must be changed. For a decrease of 1 mass unit, $\mathrm{z}^{2} / \mathrm{A}$ increases by about 0.17 in this region of the periodic table.

Thus, for cach neutron assumed to be emitted before fission, each point in Fig. 6 must be displaced along a line whose slope is

$$
6\left(\frac{\widetilde{s}_{\text {eff }}}{\Im_{0}}\right)\left(\frac{4}{E} *+0.01\right)
$$


Fór. the six points corresponding to bombardment. of gold. with nitrogen ions, this quantity (averaged over the data) is 0.4 , to be compared with a slope of the solid line of 0.2 in this region. Clearly, if neutrons are emitted before fission, the data in Fig. 6 should be displaced upwards and to the right relative to the solid curve. If there are many neutrons before fission, such agreement as there is between our results and those of chaudhry et ai. 3 would disappear. On the basis of these data, we conclude that an average of only one or two neutrons at the most are emitted before fission. Information available on the relative probabilities for fission and neutron emission suggests that for systems such as $\mathrm{Bi}^{209}$ the average number of neutrons emitted before fission is close to zero. ${ }^{15}$ Measurements of the cross section for fission induced by carbon ions on $\mathrm{Au}^{197}$ lead to the conclusion that an unknown but nonzero number of neutrons is emitted before fission. ${ }^{23}$

\section{B. The Moment of Inertia}

The moment of inertia of a spherical shell of radius $r$, thickness $d r$, and density $\rho$ is

$$
(8 / 3) \pi r^{4} d r
$$

and for a body with spherical symmetry is

$$
\therefore \frac{8 \pi}{3} \cdot \int_{0}^{\infty} r^{4} \rho d r
$$

The mean square radius of such a body is 


$$
\left\langle\mathrm{R}^{2}\right\rangle=\int_{0}^{\infty} \mathrm{r}^{4} \rho \mathrm{dr} / \int_{0}^{\infty} r^{2} \rho \mathrm{dr} .
$$

The mass $M$ is given as.

$$
\therefore M=4 \pi \int r^{2} \rho d r
$$

Combining the last three equations, we find that $\mathfrak{F}_{0}$, the moment of inertia of a body with spherical symmetry. is given by

$$
\Im_{0}=\frac{2}{3} M \cdot\left\langle R^{2}\right\rangle
$$

If we assume that the mass density has the same dependence on radius as the charge distribution; then the correct value of $\left\langle R^{2}\right\rangle$ to use in Eq. (A5) is that determined by the electron-scattering experiments. 27 Hofstadter shows that for mass numbers greater than about 100, the mean-square radius can be expressed to a good approximation as

$$
\left\langle R^{2}\right\rangle=\frac{3}{5} \cdot R_{u}^{2}
$$

where $R_{u}=1.2 A^{I / 3}$ fermis. Combining this with Eq. (A5), we find

$$
\Im_{0}=\frac{2}{5} \mathrm{MR}_{u}^{2}
$$

exactly the same as for a sphere of uniform density and radius equal to $1.2 \mathrm{~A}^{1 / 3}$ fermis. We have used expression (A6) in calculating our moments of inertia. 
A possible explanation for the very low moments of inertia found in these experiments for the lowest bombarding energies might be that at these low excitation energies the moment of inertia of the nucleus is less than that of a rigid body. However, the excitation energies in question are approximately the same as those encountered by Chaudhry et al., who found that they could account for their results by using rigidebody moments. 


\section{REFERENCES}

* Work done under the auspices of the U.S. Atomic Energy Commission.

†Present address: Frick Chemical Laboratory, Princeton, New Jersey.

F Present address: U.S. Atomic Energy Commission, Washington 25, D.C.

1. I. Halpern and C. T. Coffin, Phys. Rev. 1ㅡ, 536 (1958).

22. R. Vandenbosch, H. Warhanek, and J. R. Huizenga, Phys. Rev. $1244,8166(196 \perp)$.

3. R. Chaudhry, R. Vandenbosch, and J. R. Huizenga, Phys. Rev. 126, 220 (1962).

4. Proceedings of the Second Conference on Reactions Between Complex Nuclei (John Wiley and Sons, Inc., New York, 1960),pp. 201-235.

5. G. E. Gordon, A. E. Larsh, T. Sikkeland, and G. T. Seaborg, Phys. Rev. $1201341(1960)$.

6. H. C. Britt and A. R. Quinton, Phys. Rev. 120, 1768 (1960).

7. A. Bohr, in Proceedings of the International Conference on the Peaceful Uses of Atomic Energy, Vol. 2, (United Nations, New York, 1956), p. 151.

8. I. Halpern and V. Strutinski, in Proceedings of the Second United Nations International Conference on the Peaceful Uses of Atomic Energy, Vol. I5, (United Nations, Geneva, 1958), p. 408.

9. J. J. Griffin, Phys. Rev. 116, 107 (1959).

10. T. Sikkeland, E. L. Haines, and V. E. Viola, Jr., Phys. Rev. 125, 1350 (1962).

11. E. L. Hubbard, W. R. Baker, K. W. Ehlers, et al., Rev. Sci. Instr. 32, 621 $(1961)$

12. L. C. Northcliffe, Phys. Rev. 120, 17,44 (1960).

13. R. M. Sternheimer, Phys. Rev. 115, 137 (1959).

14. R. B. Leachman, in Proceedings of the Second United Nations International Conference on the Peaceful Uses of Atomic Energy, Vol. 15 (United Nations, Geneva, 1958), p. 229. 
15. J. R. Huizenga and R. Vandenbosch, Nuclear Fission, to be published in Nuclear Reactions, Vol. 2 (North Holland Publishing Co., Amsterdam).

16. A. G. W. Cameron, A Revised Semi-Empirical Mass Formula, Chalk River Report CRP-690, March 1957.

17. J. R. Huizenga, R. Chaudhry, and R. Vandenbosch, Phys. Rev. 126, 210 (1962).

18. J. R. Huizenga and G. Igo, Nucl. Phys。 29, 462 (1962).

19. F. Everling, L. A. Konig, J. H. E. Mattauch, and A. H. Wapstra, Nuclear Phys. 18, 529 (1960).

20. T. D. Thomas, Phys. Rev. 116, 703 (1959).

21. Victor E. Viola, Jr., and Torbjфrn Sikkeland, Total Cross Sections for Fission of $\mathrm{U}^{238}$ Induced by $\mathrm{He}^{4}$ and Heavy Ions, Lawrence Radiation Laboratory Report UCRL-10088, Feb. 1962 (unpublished).

22. Torbjørn Siklseland and Victor I. Viola, Jr. (Lawrence Radiation Laboratory), unpublished data.

23. T. D. Thomas, G. E. Gordon, R. M. Latimer, and G. T. Seaborg, Phys. Rev. to be published.

24. H. C. Britt and A. R. Quinton, Phys. Rev. 124, 877 (1961).

25. J. M. Alexander and L. Winsberg, Phys. Rev. 121, 529 (1961).

26. S. Cohen and W. J. Swiatecki, The Deformation Energy of a Charged Drop. IV. Evidence for a Discontinuity in the Conventional Family of SaddlePoint Shapes, Institute of Physics Report, University of Aarhuus, Aarhuus, Denmark (1961) unpublished.

27. R. Hofstadter, Ann. Rev. Nuclear Sci. I, 308 (1957). 
This report was prepared as an account of Government sponsored work. Neither the United States, nor the Commission, nor any person acting on behalf of the Commission:

A. Makes any warranty or representation, expressed or implied, with respect to the accuracy, completeness, or usefulness of the information contained in this report, or that the use of any information, apparatus, method, or process disclosed in this report may not infringe privately owned rights; or

B. Assumes any liabilities with respect to the use of, or for damages resulting from the use of any information, apparatus, method, or process disclosed in this report.

As used in the above, "person acting on behalf of the Commission" includes any employee or contractor of the Commission, or employee of such contractor, to the extent that such employee or contractor of the Commission, or employee of such contractor prepares, disseminates, or provides access to, any information pursuant to his employment or contract with the Commission, or his employment with such contractor. 